SAEs, or AEs leading to study drug discontinuation were not seen in any of the arms, whether MTX was added or not.

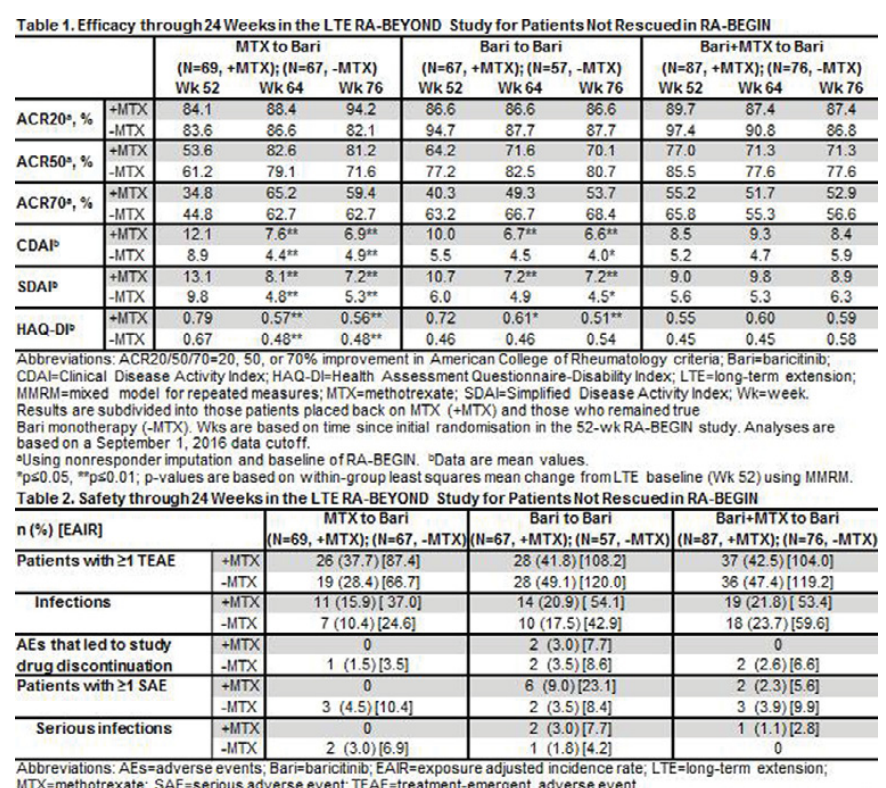

Analyses are based on a September 1,2016 data cutoff. Data are n (\%) [EAIR].

Conclusions: Switching from MTX to bari monotherapy, maintaining bari monotherapy was associated with improvements in depths of disease control during the initial 24 wks post-switch. Disease control did not significantly change after withdrawal of MTX from combination therapy. Pts who entered the LTE with suboptimal disease control after treatment with bari monotherapy may benefit from the addition of MTX. Discontinuation of MTX in pts treated with combination during the index study was associated with maintenance of response. There were no differences in important measures of safety including events that are serious or led to discontinuation.

Disclosure of Interest: R. Fleischmann Grant/research support from: AbbVie, Amgen, AstraZeneca, Bristol-Myers Squibb, Celgene, Genetech, GlaxoSmithKline, Janssen, Eli Lilly and Company, Merck, Pfizer, Regeneron, Roche, Sanofi Aventis, UCB, Consultant for: AbbVie, Akros, Amgen, Bristol-Myers Squibb, Celgene, Genentech, GSK, Janssen, Eli Lilly and Company, Pfizer, Sanofi-Aventis, UCB, T. Takeuchi Consultant for: Pfizer Japan, Astra Zeneca KK, Eli Lilly Japan KK, Novartis Pharma KK, Daiichi Sankyo Co Ltd, Nipponkayaku Ltd, Janssen Pharma KK, Merck Serono Ltd, Takeda Pharma Ltd, Mitsubishi Tanabe Pharma, Astellas Pharm, Abbvie GK, Bristol-Myers KK, Asahi Kasei Medical KK, Speakers bureau: Celtrion, Nipponkayaku Ltd, Pfizer Japan, UCB Japan, Daiichi Sankyo Ltd, Takeda Pharma Ltd, Chugai Pharma Ltd, Abbvie GK, Bristol-Myers KK, Eisai Ltd, Mitsubishi Tanabe Pharma, Janssen Pharma KK, Astellas Pharma, M. Schiff Consultant for: Abbvie, Amgen, Antares, BMS, Eli Lilly and Company, J\&J, Novartis, Novo Niordisk, Pfizer, Roche, UCB, Speakers bureau: Abbvie, BMS, D. Schlichting Employee of: Eli Lilly and Company, L. Xie Employee of: Eli Lilly and Company, M. Issa Employee of: Eli Lilly and Company, I. Stoykov Employee of: Eli Lilly and Company, J. Lisse Employee of: Eli Lilly and Company, P. Martinez-Osuna Employee of: Eli Lilly and Company, T. Rooney Employee of: Eli Lilly and Company, C. Zerbini Grant/research support from: Pfizer, Novartis, Eli Lilly and Company, Merck, Sanofi, Amgen, Celtrion, Consultant for: Merck, Pfizer, Sanofi, Eli Lilly and Company

DOI: 10.1136/annrheumdis-2017-eular.1334

\section{SAT0059 HIGH RADIOGRAPHIC PROGRESSION IS RARE AND GETS LESS FREQUENT IN RA PATIENTS OF THE SWISS SCQM COHORT FROM 1998 TO 2015}

$\underline{\text { R. Mueller }}^{1}$, R. Thalmann ${ }^{1}$, H. Schulze-Koops ${ }^{2}$, N. Graf ${ }^{3}$, J. von Kempis ${ }^{1}$. ${ }^{1}$ Division of Rheumatology, Kantonsspital St. Gallen, St. Gallen, Switzerland;

${ }^{2}$ Rheumaeinheit, Klinikum der Universität München, Campus Innenstadt, Munich, Germany; ${ }^{3}$ Graf Biostatistics, Winterthur, Switzerland

Background: The primary goal of current therapeutic strategies in RA is to control disease activity and at the same time to prevent radiographic progression. A disconnect between a missing radiographic progression and higher disease activity under treatment with biologic agents has been discussed. In general, analyses of radiographic progression in RA rather focus on radiographic nonprogression or repair and high radiographic progression in spite of therapy has, to our knowledge not been analysed in detail in the last years, neither in RCTs nor in cohort studies.

Objectives: To analyse the occurrence of high radiographic destruction and it's development in the era of biologic treatment.
Methods: We included all RA patients from the Swiss registry SCQM with at least two subsequently scored radiographs. Radiographic destruction was scored with the Ratingen erosion score and calculated by dividing the change of Ratingen scores by time ( $>6$ months) between two subsequent radiographs. To analyse high radiographic progression we looked for the height and time of the highest (peak) radiographic progression in every individual patient for the analysis. The individual peak radiographic progression was analysed in groups as change of Ratingen scores/year: $0-\leq 10,10-\leq 20,20-\leq 30,>30$ (groups $1-4$ ). The time point of the individual peak radiographic progression was analysed per group (follow up 1998 - 2015). The baseline disease characteristics were compared using standard descriptive statistics (Kruskal-Wallis or Chi-square tests).

Results: 4'033 patients were included into the analysis. 3'049 patients had a peak radiographic progression between 0 and $\leq 10$ /year, 773 between 10 and $\leq 20,150$ between 20 and $\leq 30$, and 61 of $>30$. All patient groups were within the same age range (mean: $56.5-60.5$ years). Rheumatoid factor and ACPA were more frequent in patient groups with higher peak radiographic progression (RF: 73.6, 80.0, 88.9, 90.0; ACPA: 66.8, 73.4, 74.3, 82.1, groups $1-4$, respectively). When the rate of radiographic progression before and after peak progression was analysed, $69.7 \%, 74.7 \%, 76.9 \%$, and $93.3 \%$ of the patients had a radiographic progression of $25 \%$ or lower as compared to peak progression before and $76.1 \%$, $81.8 \%, 91.1 \%$, and $93.8 \%$ after this peak progression, respectively for patients in groups 1 to 4 (Figure A).

The disease activity, as assessed by DAS 28 , was significantly higher in all patient groups before peak progression and lower thereafter (Figure $B, p<0.001$ ). Average HAQ-DI scores increased after peak radiographic progression in group 4 (Figure $C, p=0.005$ ) whereas it is stable or even decreases among the patients of the other patient groups.

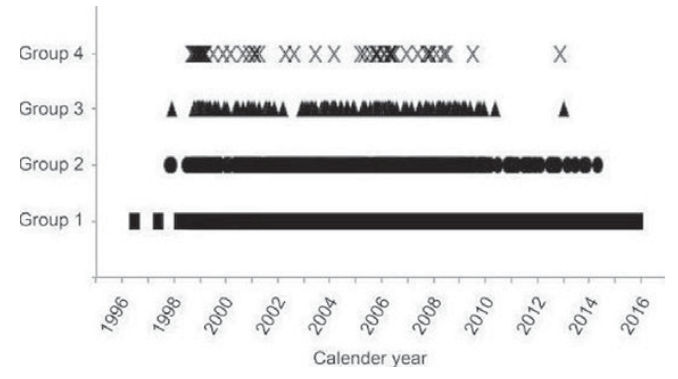

Conclusions: High peak radiographic progression is a rear phenomenon that gets less frequent in patients over the last years of observation. This may be an effect of modern therapy. Among the patients with high radiographic progression, (1) a greater delay between diagnosis and first symptoms was found and (2) the use of biologic agents was less frequent. These data suggest that the decreasing frequency of very high radiographic peak progression in the Swiss RA patients reflect a more effective therapeutic approach in the last years.

Disclosure of Interest: None declared

DOI: 10.1136/annrheumdis-2017-eular.4232

\section{SAT0060 RESPONSE TO CONVENTIONAL SYNTHETIC DMARDS DIFFERS DEPENDING ON RHEUMATOID FACTOR LEVELS IN ANTI-CITRULLINATED POSITIVE PATIENTS WITH EARLY RHEUMATOID ARTHRITIS}

S. Bugatti, A. Manzo, G. Zanframundo, F. Benaglio, G. Sakellariou, C. Montecucco, R. Caporali. Rheumatology and Translational Immunology Research Laboratories (LaRIT) and Early Arthritis Clinic, Division of Rheumatology, University of Pavia, IRCCS Policlinico San Matteo Foundation, Pavia, Italy

Background: In rheumatoid arthritis (RA), several autoantibody characteristics, including specifities, levels and isotypes, may influence their pathogenic properties and impact on clinical presentation and outcomes of the disease $(1,2)$. In particular, classical IgM rheumatoid factor (RF) may boost inflammation triggered by anti-citrullinated protein antibodies (ACPA) (3)

Objectives: To investigate whether RF impacts on disease characteristics and response to therapy in ACPA-positive early RA patients treated with conventional synthetic disease modifying drugs (csDMARDs)

Methods: 574 early RA patients consecutively enrolled in our Early Arthritis Clinic between 2005 and 2014 were included. Patients had symptoms' duration $<12$ months, were glucocorticoid- and DMARD-naïve, and fulfilled RA criteria at inclusion. IgM RF and IgG ACPA were determined in baseline sera by immunonephelometry and a second-generation EliA CCP assay respectively. Autoantibody levels were considered high when $>3$ times the upper limit of normal (ULN). Patients were treated with incremental doses of methotrexate according to a treat-to-target strategy aiming at low disease activity (LDA, DAS28 $\leq 3.2$ ). The associations between autoantibody specifities and levels and the achievement of LDA and disease remission (DAS28<2.6) over 6 months were investigated by Cox regression.

Results: 360 patients tested RF and/or ACPA positive (31.9\% single RF-positive, $10.3 \%$ single ACPA-positive, $57.8 \%$ ACPA and RF double-positive) and were 